\title{
Li-Functionalized Carbon Nanotubes for Hydrogen Storage: Importance of Size Effects
}

\author{
Ekaterina Anikina $^{\dagger, \dagger}$, Amitava Banerjee ${ }^{*}$, Valery Beskachko ${ }^{\ddagger}$, Rajeev Ahuja ${ }^{\dagger, \perp}$ \\ ${ }^{\dagger}$ Materials Theory Division, Department of Physics and Astronomy, Uppsala University, Box 516, 75120, Sweden \\ $¥$ Institute of Natural Sciences and Mathematics, South Ural State University, 76, Lenin Prospekt, Chelyabinsk 454080, \\ Russia \\ ${ }^{\perp}$ Applied Materials Physics, Department of Materials and Engineering, KTH Royal Institute of Technology, S-100 44 \\ Stockholm, Sweden
}

Corresponding Author:

*(AB) E-mail: amitava245@gmail.com

$\underline{\text { Supplementary figures }}$
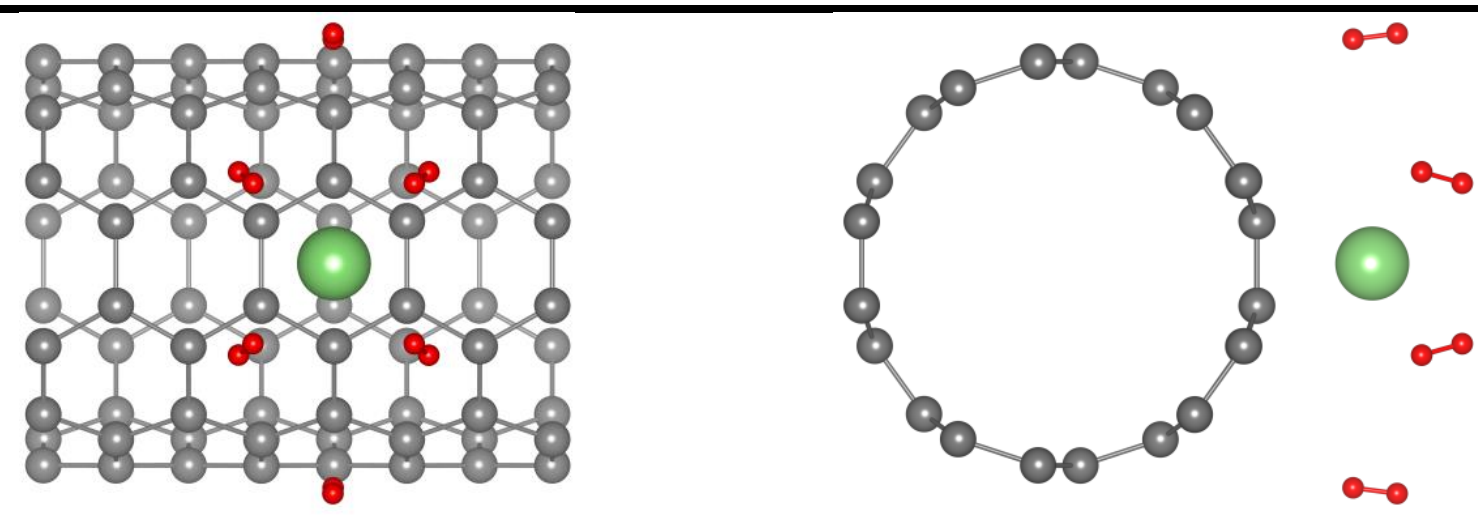

Figure S1. Configuration S+2 of the complex CNT(5,5)@ Li+6 $\mathrm{H}_{2}$ (external sorption, $\left.N_{\mathrm{c}}=4\right)$. C, Li, and $\mathrm{H}$ atoms are gray, green, and red, respectively. 

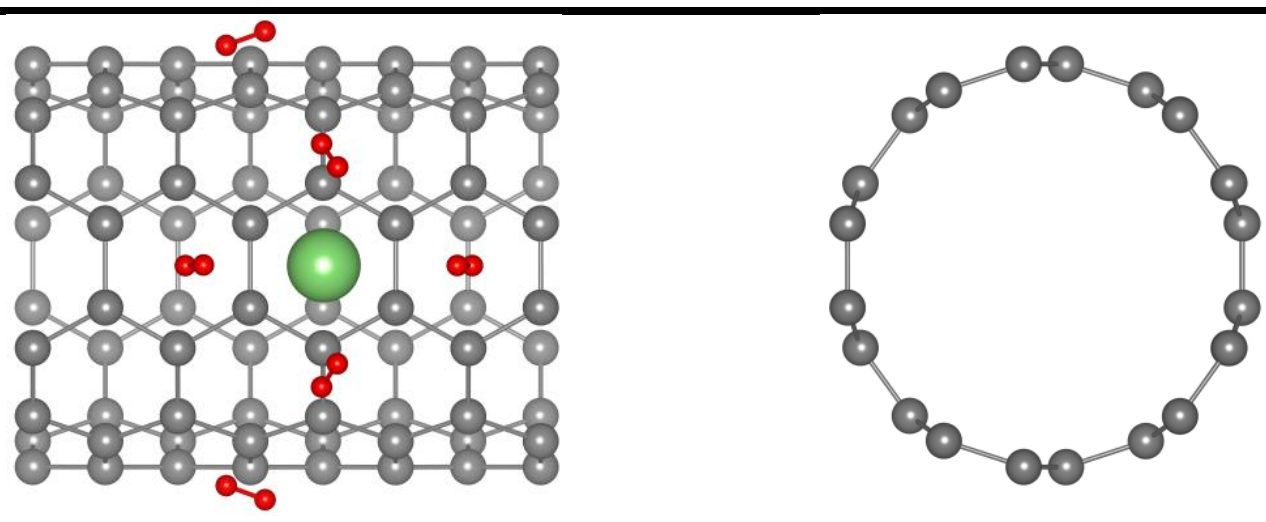

8
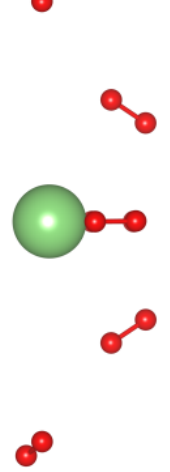

Figure S2. Configuration $\mathrm{R}+2$ of the complex CNT $(5,5) @ \mathrm{Li}+6 \mathrm{H}_{2}$ (external sorption, $\left.\mathrm{N}_{\mathrm{c}}=4\right)$. $\mathrm{C}$, Li, and $\mathrm{H}$ atoms are gray, green, and red, respectively.

a)

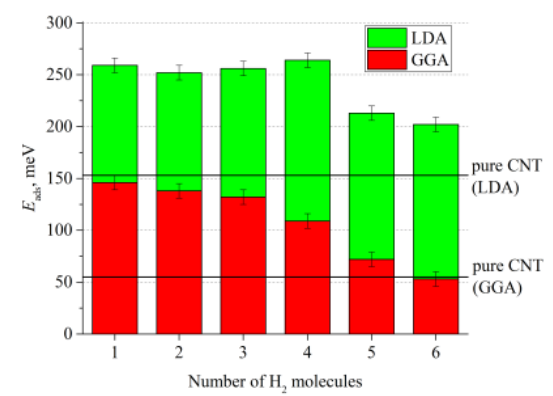

b)

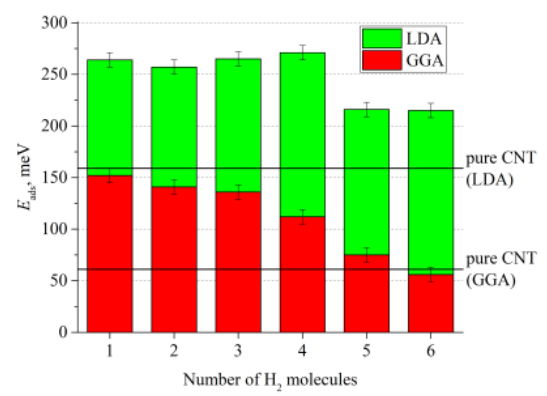

c)

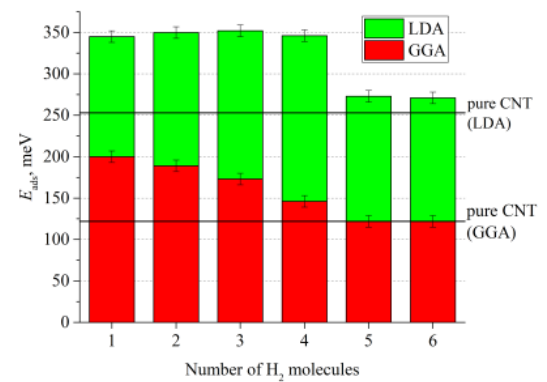

Figure S3. Hydrogen adsorption energies in the case of (a) external sorption on CNT(7,7)@Li; (b) external sorption on CNT(9,9)@Li; (c) internal sorption on CNT(9,9)@Li. GGA and LDA results are noted by red and green color, respectively. Adsorption energies on pure CNTs are noted by solid lines (for both GGA and LDA calclations).

\section{Supplementary tables}

Table S1. Ebind $(\mathrm{meV})$ of $\mathrm{H}_{2}$ Molecule in Complexes CNT(7,7)+kH 2 and CNT(7,7)@ Li+kH $\mathrm{k}_{2}$ Calculated in GGA, DFT-D2, and LDA

\begin{tabular}{|c|c|c|c|c|c|c|c|c|c|c|c|c|}
\hline \multirow{3}{*}{$k$} & \multicolumn{6}{|c|}{ CNT(7,7)@Li } & \multicolumn{6}{|c|}{$\operatorname{CNT}(7,7)$} \\
\hline & \multicolumn{3}{|c|}{ external surface } & \multicolumn{3}{|c|}{ internal surface } & \multicolumn{3}{|c|}{ external surface } & \multicolumn{3}{|c|}{ internal surface } \\
\hline & GGA & DFT-D2 & LDA & GGA & DFT-D2 & LDA & GGA & DFT-D2 & LDA & GGA & DFT-D2 & LDA \\
\hline 1 & 146 & 269 & 259 & 218 & 393 & 379 & 55 & 114 & 153 & 140 & 252 & 287 \\
\hline 2 & 143 & 264 & 252 & 204 & 378 & 386 & - & - & - & - & - & - \\
\hline 3 & 126 & 247 & 256 & 189 & 370 & 373 & - & - & - & - & - & - \\
\hline 4 & 85 & 203 & 264 & 155 & 282 & 367 & - & - & - & - & - & - \\
\hline
\end{tabular}

